\title{
Identification of Two Novel Frameshift Mutations in Exostosin 1 in Two Families with Multiple Osteochondromas
}

\author{
Chen-Yu Wang a, b, c Fang Yu ${ }^{b}$ Jie-Yuan Jin ${ }^{a}$ Ji-Qiang He ${ }^{b}$ Liang-Liang Fan ${ }^{a, c}$ \\ Ju-Yu Tang ${ }^{b}$ Rong Xiang ${ }^{a, b, c}$ \\ aDepartment of Cell Biology, School of Life Sciences, Central South University, Changsha, China; ${ }^{b}$ Department of \\ Orthopedics, Xiangya Hospital of Central South University, Changsha, China; 'Hunan Key Laboratory of Animal \\ Models for Human Diseases, School of Life Sciences, Central South University, Changsha, China
}

\section{Keywords}

EXT1 · Mutation · Long bone deformation .

Multiple osteochondromas

\begin{abstract}
Multiple osteochondromas (MO) is an autosomal dominant hereditary disorder, which typically manifests as skeletal dysplasia, mainly involving long bones and knees, ankles, elbows, wrists, shoulders, and pelvis. Previous studies have demonstrated that mutations in exostosin glycosyl transferase-1 (EXT1) and exostosin glycosyl transferase-2 (EXT2) were the main cause of $\mathrm{MO}$. In this study, we enrolled 2 families with MO. Sanger sequencing revealed 2 novel frameshift mutations - c.1432_1433insCCCCCCT; p.Lys479Profs*44 and c.1431_1431delC; p.S478PfsX10 - in the EXT1 gene detected in 2 families, respectively. Both novel mutations, located in the conserved domain of EXT1 and predicted to be disease causing by informatics programs, were absent in our 200 control cohorts and other public databases. Our study expanded the spectrum of EXT1 mutations and contributed to genetic diagnosis and counseling of patients with $\mathrm{MO}$.
\end{abstract}

(c) 2021 S. Karger AG, Basel (c) 2021 S. Karger AG, Basel

www.karger.com $/ \mathrm{ms}$

Karger $\stackrel{2}{=}$

\section{Introduction}

Multiple osteochondromas (MO), also called hereditary multiple exostoses, is an inherited bone disorder, characterized by the presence of multiple benign cartilage-capped tumors, localized primarily in the long tubular bones, particularly in the humerus (10-50\%), forearm (39-60\%), knee (33\%), and ankle (25\%) [Wu et al., 2018; Chen et al., 2019]. The estimated prevalence of MO is 1 in 50,000; moreover, the approximate ratio of its morbidity between men and women is 3:2 [Hong et al., 2017; Xu et al., 2017; Yang et al., 2019]. The patients with MO often suffer from pain due to nerve and vascular compression, and $1-2 \%$ of patients may progress into osteosarcoma or chondrosarcoma, which is one type of malignant tumor and may threat to the health and lives of patients [Hong et al., 2017; Li et al., 2018].

Previous studies have revealed that MO is an autosomal dominant hereditary disorder with 2 main diseasecausing genes identified, exostosin glycosyl transferase-1 (EXT1) and exostosin glycosyl transferase-2 (EXT2) [Santos et al., 2018; Chen et al., 2019; Oliver et al., 2019].

C.-Y.W. and F.Y. contributed equally to this work. 
Approximately $85-95 \%$ of the MO patients harbor variants of these 2 genes, but patients with EXT1 pathogenic variants were found to have a greater number of exostoses, a greater incidence of limb malalignment with shorter limb segments and height, and more frequent pelvic and flat bone involvement than those with EXT2 pathogenic variants [Pacifici, 2017; Piombo et al., 2018].

In China, the studies and investigations of $\mathrm{MO}$ are still lacking. In this study, 2 families with MO were enrolled and Sanger sequencing of EXT1 and EXT2 was performed to detect the genetic lesion of these 2 family members [Pacifici, 2018; Santos et al., 2018].

\section{Materials and Methods}

\section{Subjects}

Blood was collected from the probands and their family members; individuals were examined by X-rays.

\section{DNA Extraction}

Genomic DNA was prepared from peripheral blood of the patients and all other participants using a DNeasy Blood \& Tissue Kit (Qiagen, Valencia, CA, USA) as described [Fan et al., 2015].

\section{Polymerase Chain Reaction}

All the coding exons of the causative gene were amplified by PCR in $25 \mu \mathrm{L}$ reaction mixture, which consisted of $2 \times$ Power Taq PCR MasterMix (Bioteke corporation, PR1702) and $50 \mathrm{ng}$ of genomic DNA. The thermal cycling consisted of an initial denaturation at $95^{\circ} \mathrm{C}$ for $4 \mathrm{~min}$, followed by 35 cycles of amplification consisting of denaturation at $95^{\circ} \mathrm{C}$ for $1 \mathrm{~min}$, primer annealing at $55-61^{\circ} \mathrm{C}$ for $30 \mathrm{~s}$, and primer extension at $72^{\circ} \mathrm{C}$ for $1 \mathrm{~min}$. A final extension step was performed at $72^{\circ} \mathrm{C}$ for $7 \mathrm{~min}$.

\section{Mutation Sequencing}

The entire coding regions, including the flanking intronic sequences of EXT1 (NM_608177) and EXT2 (NM_608210), were amplified with polymerase chain reaction (PCR; primer sequences can be provided upon request). Sequences of the PCR products were determined by the ABI 3100 Genetic Analyzer (ABI).

\section{Bioinformatics Analysis}

The multiple EXT1 protein sequences across mammals were aligned using the program MUSCLE (version 3.6, an online program at http://www.ncbi.nlm.nih.gov). The MutationTaster, SIFT, and PolyPhen-2 were used to predicted the effect of the variant. The $1000 \mathrm{G}$, dbSNP132, ExAC, and GenomAD database were used to exclude the polymorphism sites.

\section{Results}

\section{Family 1}

Family 1 from Central-South China (Hunan Province) with 5 members across 3 generations participated in the study (Fig. 1a). The proband (III:1), an 11-year-old boy, presented with osteochondromas in the femur, tibia, and fibula (Fig. 1b). Simultaneously, the proband suffered from long bone deformation, and an osteotomy was performed to correct the deformation (Fig. 1b). In addition, similar symptoms were also found in his mother (II:2) and grandmother (I:4), according to the description of his father.

Sanger sequencing identified a novel insertion , c.1432_1433insCCCCCCT; p.K479PfsX44, in EXT1 in the proband (Fig. 1c). This mutation was also found in the mother of proband (II:2), but not found in another family member (II:1). Unfortunately, since the proband's grandmother has passed away, we cannot be sure whether she carried the mutation. Co-separation analysis found that this mutation was present in affected individuals and absent in healthy members. This novel mutation, resulting in a premature stop codon at position 523 in exon 6 of the EXT1 gene, was absent in our 200 healthy control cohorts as well as the 1000 Genomes project $(1000 \mathrm{G}$, www.1000genomes.org), dbSNP132 (http://www.ncbi. nlm.nih.gov/projects/SNP/), ExAC (http://exac.broadinstitute.org/), and GenomAD database (https://gnomad. broadinstitute.org/). Further bioinformatics analysis revealed that this mutation is located in a conserved site of the third extracellular domain (Fig. 1d) and is predicted to be deleterious by 3 different algorithm-based bioinformatics programs. According to American College of Medical Genetics and Genomics (ACMG) guidelines, this mutation belongs to PVS1. We consider the mutation to be highly pathogenic. Genetic analysis indicated that this insertion, c.1432_1433insCCCCCCT; p.Lys479Profs*44, in EXT1 was the genetic lesion of Family 1.

\section{Family 2}

In Family 2 (Fig. 2a), only the proband (II:2), a 17-yearold boy, presented with osteochondromas in femur, tibia, and fibula and suffered from long bone deformation (Fig. 2b). An osteotomy was performed to correct the deformation (Fig. 2b). Both his mother and father were healthy.

Mutation detection found a de novo mutation, c.1431_1431delC; p.S478PfsX10, in EXT1 present in the patient (Fig. 2c). The novel deletion resulting in a premature stop codon at position 488 in exon 6 of the EXT1 gene was also absent in our 200 healthy control cohorts as well as the 1000 Genomes project, dbSNP132, ExAC, and GenomAD database. Bioinformatics analysis revealed that this mutation is located in a conserved site of the third extracellular domain (Fig. 2d) and is predicted to be del- 


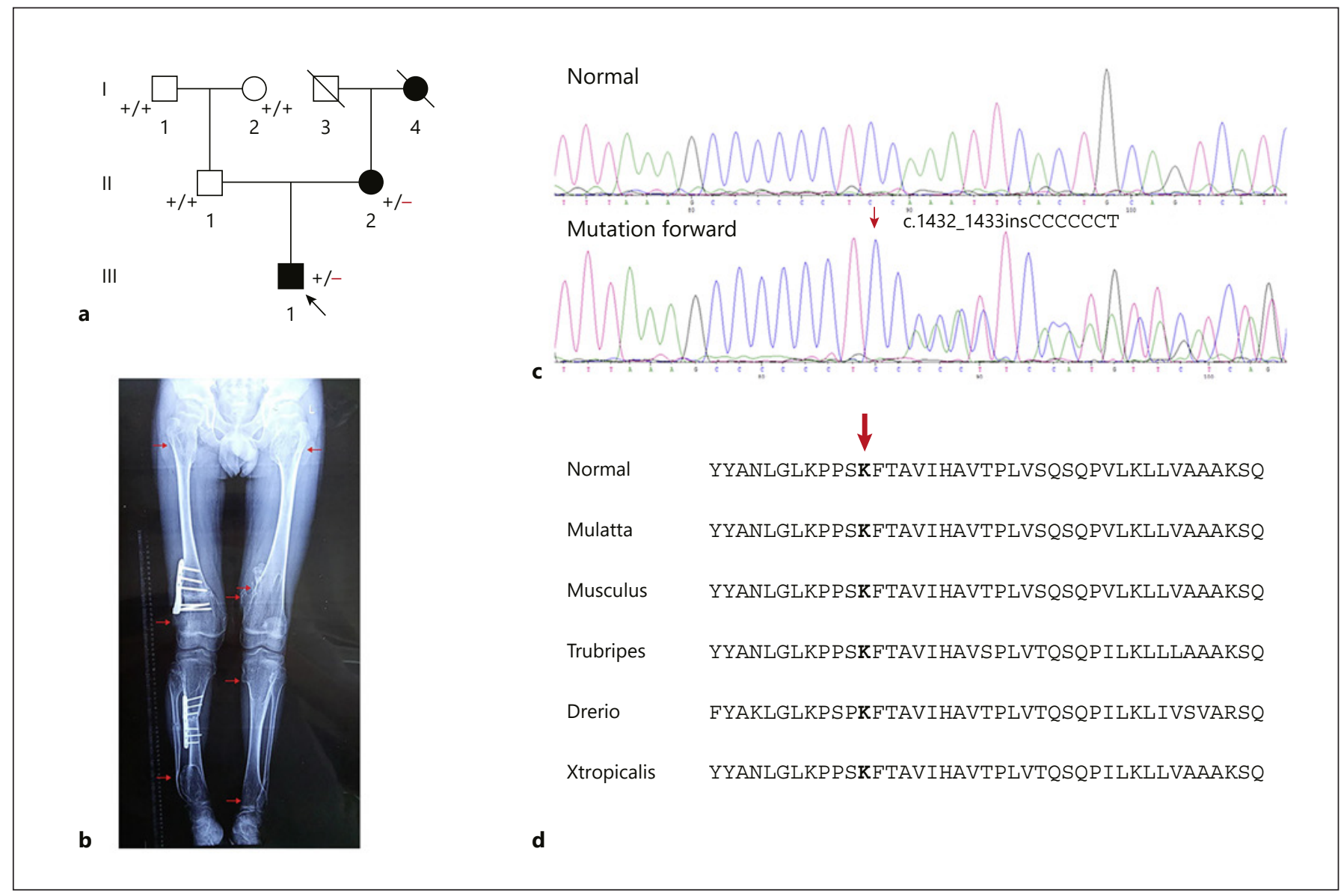

Fig. 1. a Pedigree of the family affected with MO. Family members are identified by generations and number. Squares indicate male family members; circles, female members; a closed symbol, the affected member; open symbols, unaffected members; arrow, proband. b Radiographs of proband, osteochondromas in the femur, tibia. c Sequencing result of the EXT1 mutation. A novel heterozygous frameshift mutation was detected in the exon 6 of the EXT1 gene and defined as c.1432_1433insCCCCCCT; p.K479PfsX44; d Comparison of the functional domains of EXT1 proteins encoded by mutated and normal EXT1 genes.

eterious by bioinformatics programs. According to ACMG guidelines, this mutation belongs to PVS1. We consider the mutation to be highly pathogenic. Genetic analysis indicated that this deletion, c.1431_1431delC; p.S478PfsX10, in EXT1 was the genetic lesion of the patient.

\section{Discussion and Conclusion}

The human EXT1 gene encoding exostosin glycosyl transferase-1 protein is located at chromosome 8q24.11, and it consists of 11 exons, spanning approximately 31.7 $\mathrm{kb}$ [Liang et al., 2020]. As a tumor-suppressor gene, loss of function or dysregulation of this may lead to MO and chondrosarcoma. In this study, we identified 2 novel frameshift mutations - c.1432_1433insCCCCCCT; p. K479PfsX44 and c.1431_1431delC; p.S478PfsX10 - in EXT1 in 2 families with MO. Our study was consistent with previous studies that pathogenic mutations in EXT1 may lead to MO. In addition, an osteotomy was performed to correct the long bone deformation.

EXT1 contains 2 crucial domains: the exostosin domain (aa110-396) and the glycosyltransferase domain (aa480-729) [Bai et al., 2020]. Previous studies have proven that EXT1 is a tumor-suppressor gene and the C-terminus glycosyltransferase domain of EXT1 may play a critical role in heparan sulfate biosynthesis, which is the necessary signaling of fibroblast growth factors, vascular endothelial growth factor, and transforming growth 


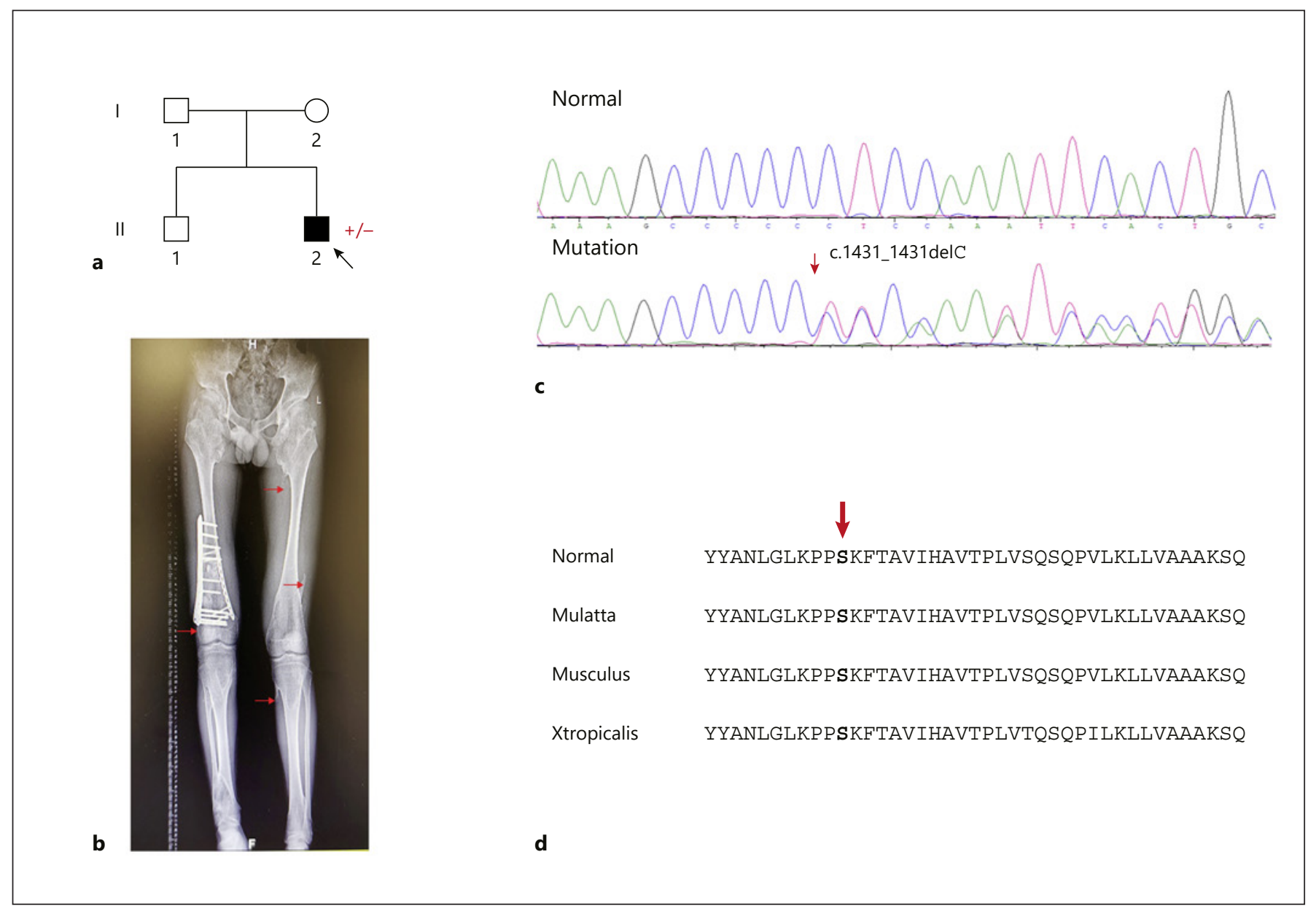

Fig. 2. a Pedigree of the family affected with MO. Family members are identified by generations and number. Squares indicate male family members; circles, female members; a closed symbol, the affected member; open symbols, unaffected members; arrow, proband. b Radiographs of proband, osteochondromas in the femur, tibia. c Sequencing result of the EXT1 mutation. A novel heterozygous deletion mutation was detected in the exon 6 of the EXT1 gene and defined as c.1431_1431delC; p.S478PfsX10; d Comparison of the functional domains of EXT1 proteins encoded by mutated and normal EXT1 genes.

factor- $\beta$, as well as the gradient formation of morphogens such as hedgehog or bone morphogenetic proteins [Sethi et al., 2019; Yang et al., 2019]. In our study, both novel mutations result in a premature stop codon at C-terminus glycosyltransferase domain, which may induce nonsense-mediated mRNA decay and lead to haploinsufficiency of the glycosyl transferase. The reduced level of glycosyl transferase may affect biosynthesis of heparan sulfate, which may disrupt the downstream signaling pathways such as bone morphogenetic proteins and result in the development of osteochondroma.

In 1995, a 1-bp deletion in the EXT1 gene was identified and co-separated in 2 of 23 unrelated families with MO [Liu et al., 2019; Oliver et al., 2019]. Since then, more than 500 mutations have been identified in patients with MO. In addition, some reports also revealed that mutations in EXT1 can cause chondrosarcoma and trichorhinophalangeal syndrome[Chen et al., 2019]. Most of these 500 mutations are nonsense, frameshift, or splice-site mutations, which result in truncation, premature termination, premature degradation, and nearly complete loss of function of the EXT1 protein. In our study, both novel mutations may induce mRNA degradation and lead to haploinsufficiency of the glycosyl transferase and result in MO.

At present, the treatment of $\mathrm{MO}$ may rely on scrape and bone graft. If the $\mathrm{MO}$ patients present with limb deformities, orthopedic correction will be an effective meth- 
od [Liang et al., 2020]. In our study, both patients had long bone deformation and osteotomy was performed to correct the malformation.

In summary, we identified 2 novel mutations c.1432_1433insCCCCCCT; p.K479PfsX44 and c.1431_1431delC; p.S478PfsX10 - in EXT1 in 2 families with MO. Our study expanded the spectrum of EXT1 mutations and contributed to genetic diagnosis and counseling of patients with MO.

\section{Acknowledgement}

We thank all subjects for participating in this study.

\section{Statement of Ethics}

The study was approved by the Xiangya Hospital of the Central South University ethics committee and was performed according to the principles of the Declaration of Helsinki. Written informed consent was obtained from all participants.

\section{Conflict of Interest Statement}

The authors have no conflicts of interest to declare.

\section{Funding Sources}

This study was supported by the National Science and Technology Major Project of the Ministry of Science and Technology of China (2017ZX10103005-006), the National Natural Science Foundation of China (81970403), the Fundamental Research Funds for the Central Universities of Hunan Province (CX20190104), the Emergency Project of Prevention and Control for COVID-19 of Central South University (160260003), and the Fundamental Research Funds for Central Universities of Central South University (2019zzts228).

\section{Author Contributions}

C.-Y.W. performed the Sanger sequencing and wrote the manuscript; F.Y. enrolled the patients; J.-Y.J. and L.-L.F. performed the DNA extraction; J.-Y.T. and R.X. designed and supported the project.

\section{References}

Bai Y, Jiao Z, Liu N, Hu S, Zhao K, Kong X. Genetic analysis of five pedigrees affected with multiple osteochondromas (in Chinese). Zhonghua Yi Xue Yi Chuan Xue Za Zhi. 2020; 37(7):717-20

Chen Z, Bi Q, Kong M, Chen Y. A Novel EXT1 Mutation Identified in a Family with Multiple Osteochondromas. Genet Test Mol Biomarkers. 2019;23(4):251-4.

Fan LL, Lin MJ, Chen YQ, Huang H, Peng DQ, Xia K, et al. Novel mutations of low-density lipoprotein receptor gene in China patients with familial hypercholesterolemia. Appl Biochem Biotechnol. 2015;176(1):101-9.

Hong G, Guo X, Yan W, Li Q, Zhao H, Ma P, et al. Identification of a novel mutation in the EXT1 gene from a patient with multiple osteochondromas by exome sequencing. Mol Med Rep. 2017;15(2):657-64.

Li Y, Wang J, Tang J, Wang Z, Han B, Li N, et al. Heterogeneous spectrum of EXT gene mutations in Chinese patients with hereditary multiple osteochondromas. Medicine. 2018; 97(42):e12855.
Liang C, Wang YJ, Wei YX, Dong Y, Zhang ZC. Identification of Novel EXT Mutations in $\mathrm{Pa}$ tients with Hereditary Multiple Exostoses Using Whole-Exome Sequencing. Orthop Surg. 2020;12(3):990-6.

Liu NW, Huang X, Liu S, Lu Y. EXT1, Regulated by MiR-665, Promotes Cell Apoptosis via ERK1/2 Signaling Pathway in Acute Lymphoblastic Leukemia. Med Sci Monit. 2019;25: 6491-503.

Oliver GR, Blackburn PR, Ellingson MS, Conboy E, Pinto E Vairo F, Webley M, et al. RNA-Seq detects a SAMD12-EXT1 fusion transcript and leads to the discovery of an EXT1 deletion in a child with multiple osteochondromas. Mol Genet Genomic Med. 2019;7(3):e00560.

Pacifici M. Hereditary Multiple Exostoses: New Insights into Pathogenesis, Clinical Complications, and Potential Treatments. Curr Osteoporos Rep. 2017;15(3):142-52.

Pacifici M. The pathogenic roles of heparan sulfate deficiency in hereditary multiple exostoses. Matrix Biol. 2018;71-72:28-39.

Piombo V, Jochmann K, Hoffmann D, Wuelling M, Vortkamp A. Signaling systems affecting the severity of multiple osteochondromas. Bone. 2018;111:71-81.
Santos SCL, Rizzo IMPO, Takata RI, Speck-Martins CE, Brum JM, Sollaci C. Analysis of mutations in EXT1 and EXT2 in Brazilian patients with multiple osteochondromas. Mol Genet Genomic Med. 2018;6(3):382-92.

Sethi S, Madden BJ, Debiec H, Charlesworth MC, Gross L, Ravindran A, et al. Exostosin 1/Exostosin 2-Associated Membranous Nephropathy. J Am Soc Nephrol. 2019;30(6):1123-36.

Wu ZY, Wang Y, Wang JW, Chen YZ, Guo Y. The role of EXT1 gene mutation and its high expression of calcitonin gene-related peptide in the development of multiple exostosis. Biochem Biophys Res Commun. 2018;505(4): 959-65.

Xu Y, Kang Q, Zhang Z. Identification of mutations in EXT1 and EXT2 genes in six Chinese families with multiple osteochondromas. $\mathrm{Mol}$ Med Rep. 2017;16(4):5599-605.

Yang A, Kim J, Jang JH, Lee C, Lee JE, Cho SY, et al. Identification of a novel mutation in EXT2 in a fourth-generation Korean family with multiple osteochondromas and overview of mutation spectrum. Ann Hum Genet. 2019; 83(3):160-70. 